\title{
Chemical composition and antioxidant activity of Strobilanthes crispus leaf extract
}

\begin{abstract}
This study investigated the components present in and the total antioxidant activity of leaves of Strobilanthes crispus (L.) Bremek or Saricocalyx crispus (L.) Bremek (Acanthacea). Proximate analyses and total antioxidant activity using ferric thiocyanate and thiobarbituric acid methods were employed. Minerals content was determined using the atomic absorption spectrophotometer, whereas the water-soluble vitamins were determined by means of the UV-VIS spectrophotometer (vitamin C) and fluorimeter (vitamins B1 and B2). Catechin, tannin, caffeine, and alkaloid contents were also studied. All data were compared to the previously reported results of Yerbamate, green tea, black tea, and Indian tea. The dried leaves contained a high amount of total ash (21.6\%) as a result of a high amount of minerals including potassium (51\%), calcium (24\%), sodium (13\%), iron (1\%), and phosphorus (1\%). High content of water-soluble vitamins (C, B1, and B2) contributed to the high antioxidant activity of the leaves. The leaves also contained a moderate amount of other proximate composition as well as other compounds such as catechins, alkaloids, caffeine, and tannin, contributing further to the total antioxidant activity. Catechins of Strobilanthes crispus leaves showed highest antioxidant activity when compared to Yerbamate and vitamin E. Consumption of the leafy extract daily ( $5 \mathrm{~g} /$ day) as an herbal tea could contribute to the additional nutrients and antioxidants needed in the body to enhance the defense system, especially toward the incidence of degenerative diseases.
\end{abstract}

Keyword: Strobilanthes crispus; Nutritional composition; Antioxidants; Catechin; Total antioxidant activity 